\title{
Rezensionen
}

\section{Herkunft und Ankunft. Neue Sammelbände zu Migrantengeschichten und zur Migrationsliteratur [Sammelrezension]}

Besprochen von Karl Esselborn: München

https://doi.org/10.1515/infodaf-2020-0008

Die Literatur von Arbeitsmigranten, politischen Flüchtlingen (aus dem Osten), freien Übersiedlern und anderen Einwanderern in Deutschland wie von ihren Kindern wurde (speziell seit den 1980er-Jahren, auch mit Unterstützung des Adelbert-von-Chamisso-Preises) von der interkulturellen Literaturwissenschaft als Geschichte der Interkulturellen deutschen Literatur in zahlreichen Sammelbänden und Handbüchern (von Gino Chiellino 2000, Alois Wierlacher/Andrea Bogner 2003 bis zu Michael Hofmann/Iulia-Karin Patrut 2015 und Walter Schmitz 2018) ausführlich dokumentiert. Sie hat sich, was die Autor(inn)en und ihre Thematik betrifft, mit den historischen Entwicklungen wie dem Ende der Sowjetunion, der deutschen Wiedervereinigung, der Verselbständigung der osteuropäischen Länder und ihrem Anschluss an die EU beständig verändert. Zuletzt hatten die Kriege im Nahen Osten und speziell der syrischen Bürgerkrieg zu einer enormen Flut von Flüchtlingen geführt, die in ihrer Heimat politisch verfolgt wurden und die in den europäischen Ländern auf ein neues Leben hofften. Sie waren, etwa am Münchner Hauptbahnhof, anfangs noch mit großer Sympathie empfangen worden. Doch mit dem Höhepunkt der Immigration nach Deutschland 2015, wozu auch noch die große Zahl Osteuropäer und Afrikaner beitrug, die in der Hoffnung auf neue, bessere Lebens- und Arbeitsmöglichkeiten illegal übers Meer nach Europa fahren, wurde die Angst vor Migranten systematisch politisch genutzt und es kam bald zu der großen nationalistischen Abwehrbewegung gegen alle Ausländer und Migranten, begleitet von Nationalismus und von Fremdenfeindlichkeit. Getragen wurde sie besonders von Pegida, der AfD und den nationalistischen Rechten (bis zu Amokläufen) und unterstützt und genutzt auch von den konservativen Parteien, die für problematische neue Außengrenzen Europas und systematische Abschiebungen eintraten. Die aktuelle Situation wurde zuletzt ausführlich beschrieben vom Sachverständigenrat deutscher Stiftungen für Integration und Migration im Jahresgutachten 2019: Bewegte Zeiten: Rückblick auf die Integrations- und Migrationspolitik der letzten Jahre (online: https://www.svr-migration. de/publikationen/jahresgutachten_2019/; 18.01.2020).

Verschiedene Organisationen, die Zuwanderer unterstützt hatten, versuchten schon früh, über deren Herkunft, Schicksal, Motive und die schwierige Situation 
im neuen Land $\mathrm{zu}$ informieren und dabei geflüchtete Autoren selbst (in der ihnen noch unbekannten Sprache) zu Wort kommen zu lassen. Dazu wurden in den letzten Jahren eine ganze Reihe von Sammelbänden vorgelegt, die hier kurz beschrieben werden. Zwei erste Anthologien stellten Autoren aus verschiedenen Ländern vor, die durch vom PEN organisierte Stipendien Writers in Exile gefördert wurden, für mehrere Jahre in Deutschland untergebracht, hier auch die Möglichkeit $\mathrm{zu}$ persönlichen Kontakten und zum literarischen Schreiben bekommen sollten.

Sigfrid Gauch, Claudia C. Krauße (Hrsg.): Ein Regen aus Kieseln wird fallen. Texte aus dem Exil. (Frankfurt am Main: Brandes \& Apsel, 2009. ISBN 978-3-86099-398-9, 398 Seiten) enthält literarische Texte von 22 Autor/innen aus verschiedenen Ländern im Osten oder in Afrika und aus Kuba, die auch im Exil - in Übersetzungen in die fremden Sprache - sich äußern können und damit ohne Isolierung oder Einschmelzung das Fremde als Bereicherung hier anbieten. Themen sind allerdings weniger die Flucht oder das neue Leben in Deutschland als vor allem die erlebte Verfolgung und die schlimmen Erfahrungen im Gefängnis; mit dem Hintergrund der schwierigen und gefährlichen Situation des investigativen Journalismus und der kritischen, oppositionellen Autorinnen und Autoren z.B. in Russland (Solowkin). Oder noch weiter zurück in die katastrophale Geschichte der Minderheiten in der Sowjetunion, den Zusammenbruch in der Gorbatschow-Zeit und die neue Generation der politisch enttäuschten Jugendlichen (Swetlana Alexijewitsch). Ähnlich die Erinnerungen an die schrecklichen Kriege in Grosny nach Aufstand der Tschetschenen (Mainat Kurbanowa). Die Journalisten Itai Mushekwe, Alhierd Bacharevič und Maxwell Sibanda aus Simbabwe beschreiben (auch in ironischer Sicht) die Geschichte und das korrupte totalitäre System ihres Landes und die Verfolgung der Kritiker durch den Geheimdienst, so wie Amir Valle die Korruption bei Polizei und Militär in Kuba. Erschreckend sind die brutalen Erfahrungen in Gefängnissen (Khalil Rostamkhani), mit Verurteilungen zum Tod oder der Erhängung eines politischen Gefangenen (in Ankara: Ahmet Kahraman). Erst nach der Flucht ins Ausland (des Kurden Selim Kaya) folgt die Ruhe und langsame Annäherung im deutschen Exil, wo man sich, aus Zeit und Raum gefallen, in anderer Kultur und fremder Sprache findet, das Innerste abgetötet, keine Gefühle mehr, und Ruhe und Alleinsein braucht, um dies aufzuarbeiten (Roshanak Daryoush) und erst mit Freunden und Arbeit sich wohler fühlt, journalistische Texte über sein eigenes Land und die grausamen Erinnerungen schreibt (Itai Mushekwe, Claudia Anthony). Im Exil zerreißen die räumlichen und zeitlichen Entfernungen das Herz. Die Ablehnung und Fremdheit im deutschen Alltag, der ständige Kampf um Zugehörigkeit, die Annäherung und Abstoßung, die Physiopsychologie des Vermissens lassen erst langsam etwa durch soziale Kontakte ein Zuhause entstehen. 
Ähnlich der vier Jahre später erschienene zweite Sammelband der Writers-inExile:

Christa Schuenke, Brigitte Struzyk (Hrsg.): Fremde Heimat. Texte aus dem Exil (Berlin: Matthes \& Seitz, 2013. ISBN 978-3-88221-096-5, 391 Seiten), in dem 20 verfolgte Autorinnen und Autoren, die (aus der Ukraine, Weißrussland, Tschetschenien, Kasachstan, Sri Lanka, Iran, Syrien, Algerien, Sierra Leone, Togo, Simbabwe, Kuba und China) nach Deutschland kamen und meist hier blieben, über ihre Vertreibung, Flucht und die Ankunft im fremden Land, im Zwischenreich, in ganz unterschiedlichen Perspektiven schreiben, aber meist mehr in literarischer Form, z.T. in Romanauszügen.

Manche Autoren waren schon zuhause in die innere Emigration, ins Schreiben, in die Freiheit und Einsamkeit gegangen und haben auch im fremden Land ihre persönliche Heimat immer bei sich, aber mit einer neuen, weiteren Sicht und einem freieren Schreiben (Alhierd Bacharevič). Besonders Autorinnen (wie die Istanbulerin Pinar Selek oder die Iranerin Mansoureh Shojaee), die schon in der Kindheit die Domestizierung und Grenzen für Frauen erfuhren und die Frauenbewegung vertreten, lernen, aus ihrer Existenz herausgerissen, ein neues, fremdes Leben, Menschen und Erfahrungen kennen, wie wenn entwurzelte, unbehauste, fremde Frauen in der ganzen Welt im Exil zuhause wären, von der internationalem Solidarität der Frauen gestützt. Gegen die triste Einsamkeit im Exil setzt die tunesische Lyrikerin Najet Adouani ihre Gedichte, ihr Wort, ihr Ich. Als Gast und Unbekannter, abgeschnitten von der Welt, erzählen Autoren im Blick zurück die traurigen Geschichten elender Geflüchteter und ihrer zerstörten Familien (Sanath Balasooriya aus Sri Lanka), beschreiben die Traditionen der Verfolgung von Intellektuellen und Schriftstellern in der Türkei (Ahmet Kahraman), in Algerien (Hamid Skif ) oder auch in Moskau (der Kasache Sergej Zolovkin). Wie Fethiye Çetin die wieder aufgetauchten Erinnerungen ihrer Großmutter an die Katastrophe der Armenier von 1915 festhält. Der in der syrischen Revolution inhaftierte kurdische Kulturkorrespondent Amer Matar schreibt ein Testament und Nachrufe aus einer Zwischenwelt auf die Märtyrerfreunde im Gefängnis wie die Getöteten. Zhou Qing aus der chinesischen Provinz erzählt detailliert die Geschichte eines studentischen Demonstranten Unter lebenden Toten in einer Gefängniszelle mit Verbrechern und Todeskandidaten. Die politischen Probleme in Simbabwe beschreibt ironisch Itai Mushekwe. Cosmos Eglo aus Togo erinnert an die großen öffentlichen Proteste nach der Tötung von zwei Studenten in Lomé und die brutale Unterdrückung der neuen Opposition. Weitere traumatische Bürgerkriegserfahrungen beschreiben die Tschetschenin Swetlana Alexijewitsch aus der zerfallenen Sowjetunion oder Maynat Kurbanova im zerstörten Grosny oder Claudia Ainajugor Anthony aus Sierra Leone und Jorge Luis Arzola sowie Amir Valle aus Kuba. 
Nach diesen Anthologien des Writers-in-Exile-Programms von nach Deutschland geflüchteten Autoren sahen sich auch deutsche Schriftsteller, Journalisten und Wissenschaftler verpflichtet, nicht nur bei Pegida-Demonstrationen öffentlich zu protestieren, sondern sich auch zu den Problemen der enormen Zuwanderungen $\mathrm{zu}$ äußern und gegen die heftigen Abwehrreaktionen und den Fremdenhass der „Patriotischen Europäer gegen die Islamisierung des Abendlandes“, Pegida, eine eigene literarische Anthologie (mit Texten von 18 Münchner Autor (inn)en, davon zwei mit Migrationshintergrund) zusammenzustellen: Schley, Fridolin (Hrsg.): Fremd. Ein Reader zum Fremden in uns und unserer Gesellschaft in Kooperation mit dem literaturportal-bayern.de (München: Kirchheim, 2015. ISBN 978-3-87410-134-9, 180 Seiten). Themen sind dabei etwa (bei Lena Gorelik) die Ängste gewöhnlicher älterer Leute auf der Straße, mit der festen Meinung, dass die Muslime überall sind, uns bedrohen und uns Deutschland wegnehmen, das uns gehört. Fremd macht Angst, und die Hierarchie der Einheimischen kennt keine Vielfalt, ist naiv und banal. Erschreckend, dass man in der Öffentlichkeit von einer Frau auf Englisch angesprochen wird, weil sie die Andere für eine Ausländerin hält und man nicht mehr weiß, wer hierher gehört (Katja Huber: Fremdschämen). Ein türkischer Name löst Vorurteile und Probleme aus, macht einen zum anderen Menschen und bringt in Kollektivverdacht (Emel Uğurcan). Thema sind auch die gefährlichen Auseinandersetzungen zwischen deutschen und „marokkanischen“ Jugendlichen (Thomas Lang) und die Probleme von schlecht (aber auch gut) informierten Jugendlichen mit Pegida-Demonstrationen (Daniel J. Kühn). Oder die Missverständnisse mit Zuwandererfamilien aus ganz anderen Kulturen (Doris Dörrie). Die Erkenntnis, dass auch bei einem offenen Gegner der Rechten in bestimmten Situationen im Alltag mit Fremden eigene Vorurteile (zu Kopftuch usw.) hervorkommen, d.h. pegide Bewusstseinsanteile der Gesellschaft (Andreas Unger: Der Pegide in mir), wird theoretisch weiter untersucht. Im Blick auf Sinnes- und Bewusstseinseindrücke, Ausländer als Fremdkörper im eigenen Bewusstsein (Steven Uhly) oder auf den problematischen Umgang der Bürokratie mit Individuen und Fremdheit (Georg Picot). Wie umgekehrt die Migration zu gesellschaftskritischer Skepsis von Wissenschaftlern wie Joseph Weizenbaum führen kann (Gunna Wendt). Andererseits entwickeln auch Deutsche Auswanderungsträume, zuletzt sogar auf den Mars (Andrea Heuser). Ein Plädoyer für mehr Mixed, für mehr Zu- und Auswanderung in aller Welt (Sandra Hoffmann) scheint besonders angebracht, wenn man schon im Dorfkindergarten muslimische türkische Kinder als ganz selbstverständlich erlebt. Und gefragt ist für die Zuwanderer auch ehrenamtliche Hilfe, etwa für eine aus Burundi geflüchtete, psychisch schwer kranke Frau, deren Abschiebung hinausgeschoben werden kann (Margarete Moulin). Wobei es auch junge Asylbewerber gibt, die schnell Deutsch lernen und gut informiert und vorbereitet sind (Christine 
Auerbach). Problematische Auswanderungswünsche lösten aber auch radikale islamische Bewegungen im Nahen Osten aus, wie die Geschichte der Wiener Mädchen bosnischer Herkunft zeigt, die zur Terrororganisation ISIS nach Syrien fuhren und als Dschihad Girls großes Aufsehen in der Presse erregten (Daniel Grohn), wozu Fridolin Schley am Ende eine eigene Dokumentation zusammengestellt hat - und er hat in Die Ungesichter (München: allitera, 2016) auch die Fluchtgeschichte einer jungen Somalierin aufgezeichnet, die heute in München lebt.

Während hier die Migrationsprobleme vor allem aus deutscher Sicht thematisiert sind, werden im folgenden aufwendigen Sammelband aus Zürich wieder Autoren vorgestellt, die in Deutschland im Exil bzw. Asyl leben: Weg sein - hier sein. Texte aus Deutschland (Zürich: Secession Verlag für Literatur, 2016. ISBN 978-3-905951-97-4, 251 Seiten; auch als mehrsprachige Ausgabe). Die (mit Portraitfotografien) vorgestellten literarischen Autorinnen und Autoren aus Syrien (17), aus Jemen und Iran (2) sollten als Betroffene an der großen Migrationsdiskussion selbst beteiligt werden und sich über Herkunft, Alltag, Flucht, neue Welt, neues Leben, Vereinsamung, Fremdheiten und Gemeinsamkeiten äußern. Ihre Texte aus Deutschland, die ihnen ihre Stimme zurückgeben, gehören zu diesem Land. Als Literatur geben sie besondere Antworten, machen uns mit Welten, mit Sichtweisen und mit uns selbst vertraut. Der deutsche Autor irakischer Herkunft Sherko Fatah, der in seinen Büchern die aktuellen Nahostkonflikte und die große Flucht beschrieben hat, betont im Vorwort, dass die literarischen Autoren auch als kleine Minderheit die Erfahrungen und Probleme der zahllosen Flüchtlinge vermitteln können, auch wenn Literatur keine biographischen Engführungen erlaubt, obwohl die private Biografie die Wahrnehmungen des Autors mit bestimmt.

Die meisten der Autor(inn)en arbeiteten in Syrien auch als Journalist(inn)en und waren erfolgreich, bis sie beim Aufstand gegen die Regierung kritisch Partei ergriffen, auch als Aktivist(inn)en für Menschenrechte, besonders auch für Frauen (Yamen Hussein, Khwala M Dunia, Rosa Yassin Hassan) oder für eine friedliche Jugend-Protestbewegung (Amer Matar), worauf sie verfolgt und z.T. eingekerkert wurden, bevor sie über die blutige Grenze (Aref Hamza) und Nachbarländer nach Deutschland fliehen konnten oder Deutschland-Stipendien von Hilfsorganisationen bekamen. Im neuen Land, einsam und isoliert, ohne Sprache, versuchen sie dann in Erzählungen, Gedichten und Romanen an ihre Herkunft und Vergangenheit und die schrecklichen Erfahrungen des Bürgerkriegs zu erinnern, die sie traumatisieren und in ständigen Alpträumen erscheinen (Raed Wahesh, Amer Matar). Der blutige Krieg (und die zunehmende Macht der salafistischen und dschihadistischen Bewegung) wird z.B. mit der Figur eines jungen Fotografen vorgeführt, der durch den Norden reist und plötzlich verschwindet 
(Mohammad Alattar). Oder die Bombardierung der Städte mit der Traumatisierung eines Jungen (Nihad Siris). In der Zeit der Proteste werden ganze Familien brutal verfolgt und getötet (Daher Ayta). Der Verlust der eigenen Welt, der Familie und Freunde und des Selbst, aller alten Begriffe wie Himmel, Liebe, der Tod in der Fremde und das Gefängnis des Exils (ohne Sprache) sind Themen von Gedichten (Khwala M Dunias, Pegah Ahmadi, Lina Atfah). Die Fremde bedeutet Warten auf ein neues Leben, das noch $\mathrm{zu}$ weit entfernt ist, einsam und verlassen (Aref Hamza), und noch länger auf ein Asyl (Noor Kanj). Anpassung misslingt sogar jungen Frauen der Freaks-Generation (Rasha Abbas). In den Zwischenräumen gibt es kein dauerhaftes Zuhause, nur ein Warten auf ein Morgen (Yamen Hussein). Aber daneben werden auch Gemeinsamkeiten im neuen Land deutlich. Die kurdische Lyrikerin Widad Nabi will in Berlin ihr früheres hartes Leben begraben oder verkaufen und sucht mit der Poesie nach Liebe. Der erfolgreiche Theatermann Ayham Majid Achan thematisiert in kurzen (ironischen) Texten Träume, Insomie, die Erwartung erfreulicher Augenblicke, die Suche nach Gerechtigkeit im Land der Ängste und des brennenden Fernseins. Und der syrisch-palästinesische Schriftsteller Ramy Al-Asheq, der in einem Flüchtlingscamp in Damaskus aufwuchs, gründet in Deutschland sogar eine arabischsprachige Zeitung. Seit ich nicht gestorben bin fühlt er sich neu geboren, ist identitätslos, genießt die Schönheit, weigert sich, in der Fremde, unterwegs auf der Suche, eine Heimat zu haben. Und das Ergebnis seiner Angst ist eine Liebe.

Was den Autor(inn)en des Bandes noch fehlt, ist allerdings das Ankommen im neuen Land und in der neuen Sprache, ein neues sich Zuhausefühlen, was sicher viel längere Zeit brauchen wird, vielleicht erst in der nächsten Generation möglich ist, wie dies die frühere Migrationsliteratur von den ,Gastarbeitern` und den Flüchtlingen aus dem sowjetischen Osten bis zu den freien Übersiedlern und ihren Kindern, den Autoren der 2. und 3. voll eingebürgerten Generation, gezeigt hat. Und auch hier erscheinen nicht die einfachen Migranten aus Nahost oder Afrika, die für neue, bessere Lebens- und Arbeitsmöglichkeiten illegal nach Europa kommen, in Flüchtlingslagern landen, wo sie oft jahrelang auf ein Asylverfahren warten und dann abgelehnt und zurückgeschickt werden oder illegal weitergehen, bzw. zuletzt an den neuen Außengrenzen und Abschiebungen scheitern. Ganz zu schweigen von den vielen zweifelhaften Einwanderern, die oft von kriminellen Gruppen hergeholt werden und für diese arbeiten müssen.

2017 erschien als Taschenbuch des Fischer-Verlags eine weitere Anthologie von Writers-in-Exile-Autoren: Josef Haslinger, Franziska Sperr (Hrsg.): Zuflucht in Deutschland. Texte verfolgter Autoren (Frankfurt am Main: Fischer, 2017. ISBN 978-3-596-29800-6, 288 Seiten) mit Texten von 20 Autorinnen und Autoren, die aus China und Georgien, aus Kuba und dem Iran, aus Syrien und anderen Ländern nach Deutschland geflohen sind. Auch sie erinnern sich an Herkunft und 
Abschied und die Ankunft im fremden Land ohne Kontakte (nach Hause), aber in einer kleinen Geborgenheit (Maynat Kurbanova aus Tschetchenien, Mansoureh Shojaee aus Iran, Sergej Zolovkin aus Kasachstan und Russland) oder in der Ratlosigkeit eines problematischen Ichs (Yamen Hussein aus Homs, Sanath Balasooriya aus Sri Lanka) und eines ungeregelten neuen Lebens (Adam Guzuev, Tschetschene). Der Stipendiat Maxwell Sibanda aus Simbabwe beschreibt dagegen ein Exotisches Deutschland in der Event- und Partyszene. Die verlorene Heimat und der Verlust ihrer selbst erscheint der tunesischen Feministin Najet Adouani in Gedanken an Großmutters Grab. Enoh Meyomesse, der in Kamerun aus der Inhaftierung freikam, thematisiert (in einem Romanauszug) die von Deutschen bestimmte Geschichte seines Landes. Die Türkin Fethiye Çetin erinnert an die Probleme der Nachkommen der Überlebenden des Völkermords an den Armeniern von 1915. Erik Arellana Bautista berichtete über die Verschwundenen im kolumbianischen Bürgerkrieg und musste 1997 für 10 Jahre nach Deutschland gehen und nach der Rückkehr später erneut. Erschreckend sind die Exilerfahrungen Bùi Thanh Hiéus, kritischer Blogger aus Hanoi, oder des ins Exil gezwungenen kubanischen Autors Amir Valle. Der Blogger Liu Dejun beschreibt seine wiederholte Verhaftung und Folterung und die ständige Überwachung von Menschenrechtlern in China. Ähnlich war Zobaen Sondhi in Bangladesch von den islamistischen Terroristen blutig verfolgt worden. Der Filmer Amer Matar entwirft ein erschreckendes (lyrisches) Bild der grausamen und unmenschlichen Niederschlagung des syrischen Aufstands. Jovan Nikolić, aus Serbien nach Deutschland emigriert, setzte sich als protestierender Lyriker, Dramatiker, Kabarettist und Songtexter für Sprache und Kultur der Roma ein. Sergej Lebedew, dem in Moskau kritische Berichte über den Ukraine-Konflikts verboten wurden, erzählt im Exil in seinen Gedichten über Krieg und Verfolgungen wie in seinen Romanen die Familiengeschichte in der Stalinzeit.

2017 hatten der Gründer der Peter-Weiss-Stiftung für Kunst und Politik e.V. und Leiter des internationalen literaturfestival berlin und die Leiterin des „Ankunft“Projekts schon auf dem 17. internationalen literaturfestival berlin einen weiteren Sammelband vorgestellt: Ulrich Schreiber, Mira Solido (Hrsg.): Ankunft. Literarische Reportagen geflüchteter Autorinnen und Autoren (Berlin: Hans Schiler, 2018. ISBN 978-3-89930-189-2, 101 Seiten). Sechs, oft mit Stipendien deutscher Stiftungen nach Deutschland gekommene syrische Autor(inn)en und drei afghanische Jugendliche sprechen (in Übersetzungen) über ihre Erfahrungen bei der Flucht und Ankunft, über den Verlust der Heimat, den oft mühsamen Erwerb der neuen Sprache, die Herausforderungen, Erwartungen, Hoffnungen und Ängste im fremden Land. Mohamad Alaaedin Abdul Moula war als frühem oppositionellen Literaten die deutsche Kultur, Philosophie und Dichtung schon vertraut, trotz der Probleme der Syrer bei den großen Unterschieden zwischen den Zivilisa- 
tionen und Kulturen, sich hier zu integrieren. Während der Theater-Autor Daher Aita sich als politischen Flüchtling in die Fremde, Zwischen zwei Welten, vertrieben sieht und sich erst langsam an Deutschland gewöhnen muss. Kheder Alagha ist das Exil - ein Leben auf Zeit, da er mit den blutigen Erinnerungen aus einem brutal niedergeschlagenen Land kam, gespalten, halb tot, nutzlos, die Kraft seines Schreibens verlor und nicht wie ein Neuankömmling leben kann. Die Lyrikerin Rasha Habbal erzählt konkreter von ihrer schwierigen Flucht mit ihrem jungen Sohn im Gummiboot nach Griechenland. Noor Kanj musste, verliebt in einem Jemeniten (mit verbotener anderer Religion, was den Verlust der Familie bedeutete), nach Europa ausreisen, über Jordanien, wo beide lange ohne Arbeit leben und sie Zwillinge abtreiben lassen muss, bevor sie unter brutalen Kontrollen und wiederholter Abweisung über die Böll-Stiftung nach Deutschland kommen, wo das schöne Leben wieder zurückkehrt und beide schließlich (sie erneut schwanger) mühsam als Flüchtlinge anerkannt werden. Es folgen kurze literarische Texte von drei afghanischen Jugendlichen, die von ihren Eltern ganz allein in einer gefährlichen Schleppertour nach Deutschland geschickt wurden, weil die geflohene Familie von Mahdi Hashemi im Iran ein elendes Leben führte und der Junge von Anwerbern des Dschihad gefährdet war, dann im neuen Land die alte Haut vermisst. Oder Kahel Kaschmiri aus dem ärmlichen ländlichen Afghanistan, den der Vater vor sexuellem Missbrauch durch Männer schützen wollte und der nach schrecklicher Bootsfahrt allein im Flüchtlingscamp in einem fremden Leben landete und erst langsam ein neues Leben findet. Sowie Shahzamir Hataki, der in Deutschland zum ersten Mal mit einem deutschen Mädchen die Erfahrung von Liebe und Verlassenwerden macht, was in Afghanistan ganz vom Familieninteresse verdeckt gewesen wäre. Weitere Gedichte der drei Jungen und von weiteren Jugendlichen sind (muttersprachlich und in deutscher und englischer Übersetzung zusammengestellt in dem Heft Allein nach Europa. Fled to Europe alone. Hrsg. von The Poetry Project e. V. (Berlin: The Poetry Project, 2017. 64 Seiten; Bezug online: https://thepoetryproject.de/gedichtband; 18.01.2020).

Als nächste Station der geflüchteten Migranten wäre das Ankommen zu verstehen, was Thema des folgenden Sammelbands ist, der die Situation von Flüchtlingen im neuen Land und den lange Prozess des Ankommens und der Loslösung von der Herkunft zum Thema macht: Katja Huber, Silke Kleemann, Fridolin Schley (Hrsg.): Wir sind hier. Geschichten über das Ankommen (München: Allitera, 2018. ISBN 978-3-96233-058-3, 224 Seiten). Das Ankommen der fremden Autoren wollte eine Gruppe Münchener Kulturschaffender mit der Begegnungsreihe Meet your neighbours, bei Treffen in Buchhandlungen oder beim Festival Acht Mal Ankommen 2018 unterstützen. Der Band enthält erzählerische und lyrische Beiträge zur Thematik von 38 Autor(inn)en aus aller Welt, auch von deutschen. Dabei wird auch die besondere Realität der Türken in Deutschland mit 
bleibendem Fremdheitsgefühl thematisiert (Banu Acun), und an Probleme im Flüchtlingslager wird aus historischer Perspektive von 1949 erinnert (Rudolf Ohlbaum). Und Sandra Hoffman verweist auf die besonders problematische Rolle der nach Albanien zurückgewiesenen Roma. Auch der internationale Wissenschaftsbetrieb spielt sich Zwischen den Orten ab, im Aufbrechen und Ankommen (Georg Picot).

Die Autor(inn)en, die meist mit Hilfe deutscher Stiftungen aus dem syrischen Kriegsinferno herauskamen, beschreiben zunächst die Situation nach dem Ankommen im neuen Land, wo man sich nun in Ruhe und Einsamkeit zuhause findet, aber das Nachdenken über die schlimmen Erfahrungen im Herkunftsland und auf der Flucht, Erinnerungen und Alpträume den Alltag bestimmen (Galal Alahmadi). Schon die Grenzüberschreitung erinnert an alte Momente des Lebens, wo etwas zu Ende ging, man sich ausliefern musste (Afraa Batous). Aber die Herkunft dominiert noch. Der Damaszener Fady Jomar gebraucht das Bild des Koffer[s], in dem Gesichter, Erinnerungen, Herkunft, Geduld, Enttäuschungen und Angst stecken, der im Schweigen zum Gefängnis und Haus wird. Aber in der Fremdheit des multikulturellen Berlin findet er Geborgenheit. Man hofft, dass die neuen Düfte die alten von Zuhause werden (Barbra Breeze Anderson aus Simbabwe). Die junge Afghanin Mariam Meetra errichtet in sich selbst ein Haus mit ihren Erinnerungen und Träumen, das sie nie verlässt, ihre Zuflucht, ihre Identität in der Einsamkeit der Fremde. Der syrisch-palästinensische Autor Ramy Al-Asheq, der schon Arabisch-deutsche Literaturtage in Berlin veranstaltete, sieht sich auch noch nicht aus dem Krieg hier angekommen, hat noch die Kälte im Kopf und das Problem mit der neuen Sprache. Selbst der Filmemacher Denijen Pauljević ist, 18 Jahre nach der Flucht aus dem Krieg nach Deutschland, kein Serbe mehr, aber auch kein Deutscher, fühlt sich wie im Märchen-Luftschloss zwischen Himmel und Erde. Und in Björn Bickers lyrischem Hohelied schwankt ein Ehepaar türkischer und brasilianischer Herkunft in Zürich zwischen Problemen und Hoffnungen. Die Damaszener Theaterfrau Rania Mleihi, die auch an deutschen Theatern erfolgreich ist, versucht sich zu entspannen, die Gefühle eines Tages und das Leben in Ruhe $\mathrm{zu}$ betrachten, um sich im neuen Land ruhig und zufrieden zu fühlen.

Die problematische Zwischenposition von jungen Zugewanderten, die in Deutschland aufwachsen, beschreibt Senthuran Varatharajah, dessen Familie 5 Jahre im Asylbewerberheim wohnte, dann in einer oberfränkischen Kleinstadt, wo man den Einheimischen eher auszuweichen versuchte. Während der deutsche Autor Friedrich Ani erzählt, wie sein Vater als Student aus Syrien nach Oberbayern gekommenen war und vor Ort zum akzeptierten Mediziner wurde. Auch Lena Gorelik hatte als junger Kontingentflüchtling aus Russland im neuen Land (zuerst im Asylantenheim) keine rechte Kindheit. Und musste, um ein neuer Mensch zu 
werden, sich nicht zu schämen, anders zu sein, sich selbst verschweigen, ihre Geschichte zurecht-legen, den Kopf erheben im Schreiben, der Sprache befehlen. Die mit ihrer uigurischen muslimischen Familie aus China eingewanderte Suli Kurban fragt sich angesichts des ständigen lästigen Fragens nach ihrer Herkunft: Wann darf ich endlich ankommen? (vgl. Mercedes Lauenstein). Dagegen sieht die im Iran-Krieg zum Studium nach Deutschland gekommene Jugendautorin Ayeda Alavie alle Menschen und Länder als Teil des großen Körpers der Menschheit und bewegt sich wie ein rotes Blutkörperchen zwischen dem deutschen und persischen Körper. Die Situation dazwischen ist etwas anders in ausländisch-deutschen Familien wie bei dem Hin und Zurück von Ariel Magnus` jüdischer Familie zwischen Deutschland und Argentinien. Oder wenn ein Afrikaner eine Familie in Deutschland gründete, aber auch noch bei der in Namibia öfter besuchten Großfamilie bleibt (Kathrin Reikowski). Während für andere Deutschland ein Neuland wird, wenn sie (wie James Tugume aus Uganda) aus einem ärmlichen Leben nach Deutschland kommen, sich verlieben und ein neues Leben finden (wie Yamen Hussein). Auch von deutschen Autoren werden etwa an afrikanischen Grenzen die Probleme der Zuwanderung gesehen (Björn Kuhligk, Andreas Unger). Annika Reich betont, dass aus deutscher Sicht ein Aufbruch aus dem Angekommensein erforderlich ist, eine Integration, nicht nur von einer Richtung aus gedacht, als notwendige Voraussetzung der geschenkten Identität in einer liberalen Demokratie. Im Gespräch mit den anderen, im Erzählen, das Nähe und Verbundenheit stiftet, kann die Angst überwunden und das neue Leben akzeptiert werden. Deutsche Autor(inn)en engagieren sich nachdrücklich für Flüchtlinge, wie Heike Geißler in Leipzig z.B. für Flüchtlingspaten, und werben um Solidarität mit Migranten wie Fridolin Schley. Die Probleme einer illegalen Einwanderung (wie von Vertretern krimineller Banden, Zuhältern und Prostituierten u.a.) spricht nur Johano Strasser an. Dass Flucht auch ein Thema für Deutsche war und ist, zeigt der Bericht Silke Kleemanns, deren Familie nach dem Krieg aus Breslau flüchten musste. Für sie war Heimat [...] etwas Genommenes, bis sie vor Ort in überraschender Vertrautheit mit Erinnerungen, gegen die Schatten der Vergangenheit, eine neue, lebendige, schöne Stadt erlebt und einen weiteren Ort der Sehnsucht und Identität gewinnt. Dagegen spielt z.B. für einen aus Südtirol nach Bayern eingewanderten alten Bauern die Grenze keine Rolle (Daniel Bayerstorfer). Ganz anders die neuen „Weltbürger“: Linda Benedikt sieht sich nach 37-maligem Ortswechsel Räumlich erweitert und fühlt sich immer mehr im Fremdsein zuhause (hat nur im Herkunftsort München noch ein Heimatgefühl). Ähnlich ist die Autorin Katja Huber überall unterwegs und sieht das Neue als das Versprechende, als sie am Meer in Coney Island einen alten Mann mit KZ-Vergangenheit trifft.

Wie wichtig und aktuell die gegenwärtige Diskussion über die Migration nach Europa war und ist, zeigt ein weiterer Band des Projekts weiterschreiben.jetzt und 
der Initiative WIR MACHEN DAS: Annika Reich, Lina Muzur (Hrsg.): Das Herz verlässt keinen Ort, an dem es hängt. Weiter schreiben - Literarische Begegnungen mit Autorinnen und Autoren aus Krisengebieten (Berlin: Ullstein, 2018. ISBN 978-3-550-05068-8, 271 Seiten). Er stellt (mit zahlreichen Bildern) ausländische Autor(inn)en aus Krisengebieten wie Syrien, Irak, Jemen und Afghanistan, dazu Sinti und Roma aus Deutschland, Österreich und Ungarn vor, um ihnen das Weiterschreiben zu ermöglichen, nachdem die Sprache wegbrach. Zugang zum deutschen Literaturbetrieb sollen ihnen literarische Tandems mit deutschen Autor(inn)en und Übersetzer(inn)en durch künstlerischen Austausch, persönliche Begegnung, Dialog und politische Diskussion geben. Eine freie Thematik umfasst neben Krieg, Vertreibung und Flucht auch andere persönliche Themen und gibt so auch den Deutschen Zugang $\mathrm{zu}$ arabischen Lebensräumen und kulturelle Traditionen. Die deutschen Autor(inn)en (oft selbst mit guten Auslandserfahrungen) beschreiben einführend ihre Kontakte mit den ausländischen Partnern und stellen diese kurz vor, deren Texte dann folgen.

Salma Salem (Pseudonym einer syrischen Autorin) wird von Saša Stanišić (dem erfolgreichen Autor aus Bosnien) vorgestellt, der bei einem Telefon-Gespräch feststellt, dass sie in Damaskus ihre Lust am Leben noch nicht verloren hat, trotz der üblen Situation, die sie beschreibt, mit den täglichen Flugzeugangriffen auf Damaskus und den vielen Toten, dem Sterben überall und den Verhaftungen in der Stadt. Annett Gröschner vergleicht ein Gedicht der syrischkurdische Schriftstellerin Widad Nabi (die auch in Berlin lebt) mit einem eigenen früheren Gedicht über ihr verschwundene[s] Haus in Ostberlin. Nabi sieht bei einem Treffen mit ihr in Prenzlauer Berg ein Haus, wo sie selbst einmal mit einem Freund lebte, einer der Orte, die sie verloren hatte, wie das Haus der Familie in Aleppo durch Krieg, Zerstörung und Abschied: aber das „Herz verlässt keinen Ort, an dem es hängt“ (33). Noor Kanj, eine Drusin aus Syrien, wird der vielgereisten Autorin Svenja Leiber bei einer Lesung mit ihrer überzeugenden Stimme vertrauter. Ihre Gedichte thematisieren die im Krieg zerstörte Stadt, den Verlust der Familie und die leeren Tage, den Kopf an falscher Stelle. Die Berliner Autorin Ulla Lenze versteht sich schnell mit Rabab Haidar (Böll-Stipendiatin aus Damaskus), die ihre schwierige Kindheit als Mädchen in einer großen syrischen Familie in der Kriegszeit auf dem Land beschreibt, dominiert von Männern und der Großmutter. In einer Laudatio der mit ihren deutschen Romanen erfolgreichen Georgierin Nino Haratischwili wird die syrische Lyrikerin Lina Atfah vorgestellt, die 2014 nach Deutschland kam (und 2018 eine Anthologie syrischer Gedichte: Deine Angst Dein Paradies mit herausgab), in deren Gedichten eine Frau und sieben deutsche Autor(inn)en gefeiert werden. David Wagner erzählt, wie er den syrischen Autor Fady Jomar in Berlin kennenlernte, der aus der Türkei (zeitweise im Gefängnis) nach Deutschland kam, wo er Asyl beantragte und ein zweites Leben als Koch 
fand. Für ihn steht der Koffer für den Halt, wo wir uns sammeln, für die problematischen und guten Erfahrungen des Geflohenen, er wird zum Gefängnis und Haus. Galal Alahmadi (in Saudi-Arabien geborener Jemenit, der sich auch in Jordanien und im Libanon immer im Exil fühlte, seit 2016 in Deutschland ist) und die Berlinerin Tanja Dückers arbeiten gemeinsam an ihren Texten. Sein Gedicht Vom Krieg beschreibt dessen Gewalt weniger lokal und direkt, zu Hause sitzt er allein am Tisch mit all seinen Erinnerungen und Alpträumen. Lena Gorelik (erfolgreiche Autorin mit Petersburger Herkunft) stellt den syrischen Lyriker Yamen Hussein vor, der über den Libanon und die Türkei nach München floh. Der Erinnerung an den Großvater im syrischen Dorf und den Assad-Krieg kann sein Gedächtnis nicht entkommen. Ali Al-Kurdi (palästinensischer Schriftsteller, der in Damaskus im Gefängnis saß, jetzt in Weimar lebt) und Martin Kordić (Sohn bosnisch-kroatischer Einwanderer, erfolgreich mit seinem ersten Herkunftsroman) kommunizieren über den Laptop, das Schreiben über ihre Vergangenheit und Zukunft verbindet sie. Die Lyrikerin Mariam Meetra (aus Pakistan, in Berlin lebend) und die in Potsdam geborene Antje Rávic Strubel teilen in einem literarischen Gespräch Erinnerungen an verlorenes Leben und Gesellschaften in Afghanistan und der DDR. Meetras Identität, ihre Träume und Ängste sind nur erhalten in ihrem innersten Haus, das seit Jahren über ihr einstürzt und die Erinnerung auch der anderen braucht. Die Geschichten Samuel Magos (aus einer Budapester Romafamilie, in Wien lebend) haben für Olga Grjasnowa (in Deutschland erfolgreiche Autorin aus Aserbaidschan, ebenfalls mit jüdischen Wurzeln) durch ihren ständigen Spagat zwischen Melancholie und Humor, Tradition und Gegenwart einen ganz alten Zauber. Er beschreibt eine traditionelle ärmliche Stadt im Osten, wo sich nicht viel änderte und die Tochter mit ihrer dunkelhäutigen Freundin nur noch zur Beerdigung der Mutter zurückkommen kann. Nora Bossong (geboren in Berlin) trifft bei einer Lesung von Autoren aus „Kriegs- und Krisenregionen“ auf der Frankfurter Messe Rasha Habbal (eine Roma aus Syrien, in Trier lebend), die versucht, eine Brücke zu bauen zwischen dem Zurückgelassenen und dem noch entfernten Land, das eine neue Heimat werden könnte. Michael Krüger bewundert Ahmad Katlesh aus Damaskus, der in Düren für Kulturmagazine, einen Soundkanal oder Theaterperformances erfolgreich ist, als neuen interkulturellen Autor. Der erinnert sich an die Kindheit in alten Lehmhäusern und sammelt die Briefe von Freunden, die sich aus seinem Leben verabschiedeten. Kristine Bilkau ist beteiligt bei einer Lesung Omar Al-Jaffals aus Damaskus, Stipendiat und Anhänger Heinrich Bölls, welchem er in einem Brief seine Situation in einer zugrunde gehenden Welt beschreibt. Antje Rávic Strubel möchte im Gespräch versuchen, der in einer schwedischen Kleinstadt lebenden syrische Theaterfrau Souad Alkhateeb beim Anschluss an das Kulturleben zu helfen, deren wütendes Schreiben im Krieg auf Deutsch erschien und die sich hier an ihre 
schwierige Pubertät auf dem Land erinnert. Monika Rinck spricht mit Ramy AIAsheq, syrisch-palästinensischem Journalisten und Lyriker, der als Stipendiat in Deutschland arabisch-deutsche Literaturtage organisierte, über ein Übersetzen seiner Gedichte, die hier seine Situation und Gefühle im fremden Land thematisieren: Den Dichtern folgt nur ihre Traurigkeit. All diese persönlichen und literarischen Gespräche und die Zusammenarbeit der hier vorgestellten literarischen Tandems tragen deutlich zur Hilfe für die Ankunft und Aufnahme der Autoren aus den Krisengebieten bei.

Ein letzter Sammelband zeigt noch eine andere, kritische Perspektive: Migrantenautor(inn)en beschreiben von ihrer Seite aus kritisch und schonungslos die tägliche Erfahrung, in Deutschland in einer rassistischen und antisemitischen Gesellschaft gelandet zu sein: Fatma Aydemir, Hengameh Yaghoobifarah (Hrsg.): Eure Heimat ist unser Albtraum (Berlin: Ullstein fünf, 2019. ISBN 978-3-96101036-3, 208 Seiten). Das Buch ist ein Manifest gegen Heimat als völkisch verklärtes Konzept, gegen dessen Normalisierung sich 14 deutschsprachige Autor(inn)en (in Beiträgen zu typischen Stichworten) durch schonungslose Perspektiven auf eine rassistische und antisemitische Gesellschaft wehren. Wenn man in Deutschland aufgewachsen ist, aber ständig als Fremde wahrgenommen wird und Probleme mit einer Assimilation hat und deshalb lieber „unsichtbar“ bleiben möchte (Sasha Marianna Salzmann), ist die „Heimat“, besonders in ihrer neokonservativen Form, ein Alptraum. Auch für die, die aus Existenzangst schlechter bezahlte Arbeit annehmen und intensiver arbeiten müssen, weil sie sich noch nicht alles nehmen können, was ihnen zusteht (Fatma Aydemir). Wenn das Land, in dem man aufgewachsen ist, sich nicht um einen schert, ja bekämpft, fehlt das Vertrauen und braucht es ein Empowerment der Marginalisierten (wie es der Journalist und Romanautor Deniz Utlu auch in seinem Magazin freitext systematisch vertritt), das in Kunst und Medien fehlt. Sharon Dodua Otoo, deren Eltern aus Ghana nach England und Deutschland kamen, beschreibt, wie ihr Sohn (mit weißem Vater) sich gegen strukturellen Rassismus in der Schule mit verbaler Selbstverteidigung wehrte und erfolgreich dafür kämpfte, sich in Berlin als Heimat wohlzufühlen. Ständige exotisierende, ent- oder hypersexualisierende Blicke (mit Fotos) zieht die junge Deutsch-Iranerin Hengameh Yaghoobifarah auf sich, die queer und als Außenseiterin mit Style auftritt, aber auch (im Iran) als weiß gesehen wird. Aber wenn man angreift, entsteht mehr Akzeptanz. Enrico Ippolito sieht sich wegen seines italienischen Namens überall der Beleidigung durch einen strukturellen, institutionellen oder alltäglichen Rassismus ausgesetzt, den die Freunde kleinreden, den er aber überall sieht, im System verankert, in der Sprache festgeschrieben. In einer rassistischen Gesellschaft aufgewachsen, sieht er sich schließlich selbst als Rassist. Für die Kulturwissenschaftlerin Mithu Sanyal ist die Frage „Wo kommst du eigentlich her?“ ein No-Go, die denen nicht gestellt 
werden kann, die hierhergehören. Im pluralistischen Einwanderungsland kann nicht auf die alten deutschen Heimat-Begriffe zurückgegriffen werden. Heimat muss in einem Prozess der Konsensbildung hergestellt werden, aus dem expliziten Verlangen, zusammenzuleben in offenen Identitäten mit geteilter Menschlichkeit und Erinnerungskultur. Mit arabischem Namen konnte Nadia Shehadeh auch als Lokalreporterin in einer deutschen Kleinstadt nicht unauffällig wie alle sein, besonders wegen der großen politischen Konflikte mit der arabischen Welt, die von den Boulevard-Medien arabisiert und islamisiert wurden, ähnlich wie männliche Fehlgriffe gegen Frauen. Sie gewöhnte sich daran, soziale Verletzungen durch Mikroaggressionen im Alltag auszublenden, während sie alle Formen von Rassismus als politische Haltung anprangert. Olga Grasnowa sieht sich als Kind jüdischer Kontingentflüchtlinge aus Russland nicht mehr als „fremd“ wahrgenommen und verweist auf eine neue Hierarchie der Migrant(inn)en oder Wirtschaftsflüchtlinge, die günstiger in anderen Ländern leben. Als Schriftstellerin sieht sie sich der (nicht biodeutschen) Migrationsliteratur (der nicht hier Geborenen oder hierher Gekommenen) zugeordnet, was sie für einen ganz fragwürdigen rassistischen und paternalistischen Begriff hält, der auch erfolgreichen Sängerinnen oder Politikerinnen Privilegien abspricht. Die „Auslandskoreanerin“ Vina Yun verweist auf die Geborgenheit, Zugehörigkeit und Gemeinschaft, die ihr das traditionelle koreanische Essen (der Mutter) verschafft (das sie zu Ausländern machte und das erst spät in Europa angenommen wurde). Margarete Stokowski betont die positive Rolle der Mehrsprachigkeit von Migrantenkindern auch im öffentlichen Raum, die auch für das (oft versteckte) Polnisch oder Türkisch gilt. Reyhan Şahin verteidigt ihr sehr offenes „Female Sexspeech“ (über ihre deutschtürkischen Freiheiten) gegen das Sex-Stigma als ,interSEXionale“ Ausgrenzungsform und das männliche Hatespeech. Der „Judenautor“ Max Czollek verweist darauf, wie „normale“ Vorstellungen von Zugehörigkeit und Homogenität und die Rückkehr rechten völkischen Denkens die Forderung nach Integration bestimmen. Für eine Integration in Deutschland werden die (meist) aus der Sowjetunion immigrierten Juden nicht aufgenommen bzw. ihre Identität wird konstruiert; die vielfältige Identität wird auf bestimmte kontrollierbare Merkmale angeblicher deutscher Normalität reduziert, statt sie als permanente Gegenwartsbewältigung, Desintegration und Selbstbestimmung in Teilhabe zu sehen. Simone Dede Ayivi setzt nicht auf Rückzug aufs Selbst und das Private, denn auf Wut und Stress im Alltag folgt erst Entspannung und Zuversicht, wenn jemand sich solidarisch zeigt. Dem Alltagsrassismus begegnet man am besten mit Alltagssolidarität, Austausch, Anteilnahme, Community-Events, dem „Zusammen“.

Abschließend wäre daran zu erinnern, dass über die neuen Formen der Migration der letzten Jahrzehnte auch schon in deutschsprachigen Romanen von Autoren geschrieben wurde, die bereits längere Zeit in Deutschland leben oder 
hier geboren sind, wovon einige der zuletzt erschienenen Bücher hier noch genannt werden sollen. Eine Sonderform war dabei Jenny Erpenbeck: „Gehen, ging, gegangen“ (München: Knaus, 2015. ISBN 978-3-8135-0370-8, 352 Seiten), wo beschrieben wird, wie ein älterer Ostberliner Professor sich von afrikanischen Flüchtlingen eines Berliner Protestcamps ihre schlimmen Lebens- und Fluchtgeschichten erzählen lässt. Eine lange gefährliche Flucht aus dem Irak nach Deutschland beschreibt Abbas Khider: Der falsche Inder (Hamburg: Edition Nautilus, 2008. ISBN 978-3-89401-576-3, 160 Seiten). Sherko Fatah: Das dunkle Schiff (Salzburg: Jung und Jung, 2008. ISBN 978-3-902497-36-9, 440 Seiten) erzählt die Geschichte eines kurdischen Jugendlichen aus dem Irak, der vor den „Gotteskriegern“, die ihn missbrauchten, nach Berlin flieht, wo ihn die radikalen religiösen Ideen wieder einholen. Julya Rabinowich: Die Erdfresserin (Wien: Deuticke, 2012. ISBN 978-3-552-06195-8, 235 Seiten) ist die etwas andere Geschichte einer jungen Frau aus Westrussland, die im Westen arbeiten möchte, aber als illegale Immigrantin in der Prostitution landet und einen Zusammenbruch hat und zuletzt beim Zurückgehen ihre Identität verliert. Olga Grjasnowa: Gott ist nicht schüchtern (Berlin: Aufbau, 2017. ISBN 978-3-7466-3439-5, 309 Seiten) thematisiert die zerstörende Flucht junger Bürgerlicher aus dem Syrien des Bürgerkriegs.

\section{Literatur}

Chiellino, Carmine Gino (Hrsg.) (2000): Interkulturelle Literatur in Deutschland. Ein Handbuch. Stuttgart: Metzler.

Hofmann, Michael; Patrut, lulia-Karin (2015): Einführung in die interkulturelle Literatur. Darmstadt: Wissenschaftliche Buchgesellschaft.

Schmitz, Walter (2018): Handbuch Literatur der Migration in den deutschsprachigen Ländern seit 1945. Band 1: ,Einwanderungsland wider Willen‘. Prozess und Diskurs. Dresden: Thelem.

Wierlacher, Alois; Bogner, Andrea (Hrsg.) (2003): Handbuch interkulturelle Germanistik. Stuttgart: Metzler. 